\title{
Replicability and reproducibility through the lens of local statistical modeling
}

Reproducibility and replicability (R\&R), long central concerns of the physical sciences, have recently become popular topics across emerging data-driven disciplines, such as geographic information science (GISc). This is likely a function of our increasing ability to accurately measure the world around us at higher spatial-temporal frequencies with relative ease and low cost compared to what was previously possible. At the same time, a brief survey of the literature in GISc does not seem to suggest distinct definitions for R\&R, with authors often using the terms with varying intent (e.g. Brundson, 2015; Singleton et al., 2016; Arribas-Bel et al., 2017; Konkal et al., 2018). This pattern can also be observed more broadly across scientific domains but some contributions from outside of GISc appear to have advocated for clearer divisions between the two terms (Plesser, 2018). On the one hand we can learn from these discussions in other fields, while on the other hand, it is important to acknowledge the unique $R \& R$ challenges and opportunities arising within GISc. Therefore, in this short position paper I utilize insights from outside of GISc as a point of departure in order to support R\&R in GISc through the lens of local multivariate statistical modeling.

One particularly vocal stance that arises out of the artificial intelligence community is that of Chris Drummond $(2009,2018)$ who contends that, "replicability is the impoverished version of reproducibility that is not worth having"(Drummond, 2009, p.2). He argues that replication and reproducibility are different beasts and that his field currently uses the term reproducibility as a misnomer for the less important property of replicability. In his view, the differences are simple; reproducibility requires changes while replicability avoids them. Similar results can be achieved with different methods and sources of data, as well as different people, labs, and equipment. The greater the difference between two experiments supporting the same conclusion, the stronger the evidence is in favor of a particular hypothesis. It follows then that replication is the weakest form of reproducibility because precisely copying an experiment and its results does not provide any new evidence. Drummond therefore supports the view that it would not be worth the time and cost of requiring research artifacts, such as shareable code and data to be submitted and reviewed during publication (2009). I argue against this view in the context of GISc through a brief synopsis of experiences within the paradigm of local multivariate statistical modeling.

Local models are naturally suited to investigate reproducibility when modeling spatial processes. They are unique in that they do not a priori assume that results are reproducible across an entire study area, which is a common implicit assumption underlying many other techniques. In particular, local multivariate statistical models explicitly measure how relationships change by spatial context and provide a framework to incorporate multiple factors and their associated uncertainties. Geographically weighted regression (GWR) (Fotheringham et al., 2002) is one such technique that has received much attention in GISc. and where replicability is essential to ongoing research.

In order to capture spatially varying processes, GWR creates subsets of samples by borrowing data that are nearby to calibration locations of interest. The extent that data are borrowed is regulated by a bandwidth parameter that can be interpreted as the scale at which a spatial relationship exists when it is selected via an optimal bias-variance tradeoff. This selection procedure and the associated GWR diagnostics require non-trivial 
amounts of code. Moreover, there are many variations of GWR that are possible through the choice of different weighting schemes. As a result, it is valuable to have open source software routines available to allow the different GWR variations to be replicated. Most notably, it increases accessibility and reduces duplication efforts. Replicable methods allow resources to be redirected towards reproducibility; a model can be confidently employed to measure the same process while varying the study area extent, data sources, or computational equipment in order to accrue evidence towards a theory. In addition, a lack of replicable code would limit discussion amongst researchers and hinder new advancements. In this sense, replicability provides a verifiable infrastructure to build upon and a baseline intuition for which to fuel knowledge discovery and production (Brundson, 2015; Singleton et al., 2016; Arribas-Bel et al., 2017).

Data access is another important aspect of replication that plays a factor in our ability to advance local statistical modeling conceptual thinking. For example, a pair of experiments examined the expected properties of surfaces (i.e., maps) of spatial processes using different local models (Griffith, 2008, Oshan \& Fotheringham, 2018). Though the experiments provide opposing views, together they've helped to clarify why different methods could lead to different conclusions. This would have been very difficult had the bespoke data employed in the original study not been made available by the author.

Another example of the importance of data fidelity in local modeling research arises from inquiries into the nature of multicollinearity in GWR (Wheeler \& Tiefelsdorf, 2005; Fotheringham \& Oshan, 2016). Though Wheeler \& Tiefelsdorf (2005) posit that the effects of multicollinearity might obfuscate GWR results, Fotheringham \& Oshan (2016) demonstrate that GWR is not uniquely susceptible to multicollinearity compared to a 'global' least squares model when the sample size is sufficiently large. More recently, a multiscale variation of GWR (MGWR) has been developed (Fotheringham et al., 2017) that allows each process represented in a model to vary at a distinct spatial scale and evidence has emerged that using a single scale of analysis can exacerbate multicollinearity (Comber et al., 2018; Oshan et al., 2018). It would therefore be interesting to gauge the extent that the results in (Wheeler \& Tiefelsdorf, 2005) were driven by the restriction to a single scale of analysis. Unfortunately, this is not possible because the original data and details about it are no longer available (Wheeler, 2018).

Counter to the Drummond's (2009) claim, replication has a useful role to play in reproducible GISc research by helping to enhance and disseminate the necessary tools. The importance of replication is perhaps amplified in GISc even more because methods developed within the discipline are applied across many domains. Consequently, a flawed result can have far-ranging impacts and it may take more time to communicate updates and new concepts. Replication efforts should therefore be pursued when possible by sharing data and adhering to open source software development practices, such as providing documentation and modular testable functions rather than custom scripts. Other helpful strategies could include new forums for recognizing results that fail to be $R \& R$ in a constructive manner and ways to reward efforts to be open and support R\&R.

Several reasons were provided in support of replicability through the availability of code and data to support local modeling research and ultimately reproducibility in GISc more broadly. Recent and ongoing research in local modeling has pursued replicability (e.g., Oshan et al., 2018) and it seems clear that without replicability, there would be far fewer sophisticated tools available to facilitate reproducibility in GISc. 


\section{References}

Arribbas-Bel, Daniel, Thomas de Graaff, and Sergio J. Rey. 2017. "Looking at John Snow's Cholera Map from the Twenty First Century: A Practical Primer on Reproducibility and Open Science" in Regional Research Frontiers Vol. 2” eds. Randal Jackson and Peter Schaeffer. Cham: Springer International Publishing.

Brunsdon, Chris. 2016. "Quantitative Methods I: Reproducible Research and Quantitative Geography." Progress in Human Geography 40 (5): 687-96. https://doi.org/10.1177/0309132515599625.

Drummond, Chris. 2009. "Replicability Is Not Reproducibility: Nor Is It Good Science.” http://cogprints.org/7691/.

Drummond, Chris. 2018. "Reproducible Research: A Minority Opinion.” Journal of Experimental \& Theoretical Artificial Intelligence 30 (1): 1-11. https://doi.org/10.1080/0952813X.2017.1413140.

Fotheringham, A. Stewart, Chris Brunsdon, and Martin Charlton. 2002. Geographically Weighted Regression: The Analysis of Spatially Varying Relationships. John Wiley \& Sons.

Fotheringham, A. Stewart, and Taylor M. Oshan. 2016. "Geographically Weighted Regression and Multicollinearity: Dispelling the Myth.” Journal of Geographical Systems, September. https://doi.org/10.1007/s10109-016-0239-5.

Fotheringham, A. Stewart, Wenbai Yang, and Wei Kang. 2017. "Multi-Scale Geographically Weighted Regression.” Annals of the American Association of Geographers 107 (6): 1247-65.

Griffith, Daniel A. 2008. "Spatial-Filtering-Based Contributions to a Critique of Geographically Weighted Regression (GWR).” Environment and Planning A 40 (11): 2751-69. https://doi.org/10.1068/a38218.

Konkol, Markus, Christian Kray, and Max Pfeiffer. 2018. "Computational Reproducibility in Geoscientific Papers: Insights from a Series of Studies with Geoscientists and a Reproduction Study." International Journal of Geographical Information Science, August, 1-22. https://doi.org/10.1080/13658816.2018.1508687.

Oshan, Taylor M., and A. Stewart Fotheringham. 2018. "A Comparison of Spatially Varying Regression Coefficient Estimates Using Geographically Weighted and Spatial-Filter-Based Techniques: A Comparison of Spatially Varying Regression.” Geographical Analysis, 50(1) 53-75. https://doi.org/10.1111/gean.12133.

Oshan, Taylor, Ziqi Li, Wei Kang, Levi Wolf, and Alexander S. Fotheringham. 2018. "Mgwr: A Python Implementation of Multiscale Geographically Weighted Regression for Investigating Process Spatial Heterogeneity and Scale."

Plesser, Hans E. 2018. "Reproducibility vs. Replicability: A Brief History of a Confused Terminology." Frontiers in Neuroinformatics 11 (January). https://doi.org/10.3389/fninf.2017.00076.

Singleton, Alex David, Seth Spielman, and Chris Brunsdon. 2016. "Establishing a Framework for Open Geographic Information Science." International Journal of Geographical Information Science 30 (8): 1507-21. https://doi.org/10.1080/13658816.2015.1137579.

Wheeler, David, and Michael Tiefelsdorf. 2005. "Multicollinearity and Correlation among Local Regression Coefficients in Geographically Weighted Regression." Journal of Geographical Systems 7 (2): 161-87. https://doi.org/10.1007/s10109-005-0155-6.

Wheeler, David. 2018. Email correspondence, May 8th 